\title{
ASSIMILATING SATELLITE SOIL MOISTURE AND FLOOD EXTENT MAPS INTO A FLOOD PREDICTION MODEL.
}

Renaud Hostache, Patrick Matgen, Peter-Jan van Leeuwen, Nancy Nichols, Marco Chini, Ramona Pelich, Carole Delenne
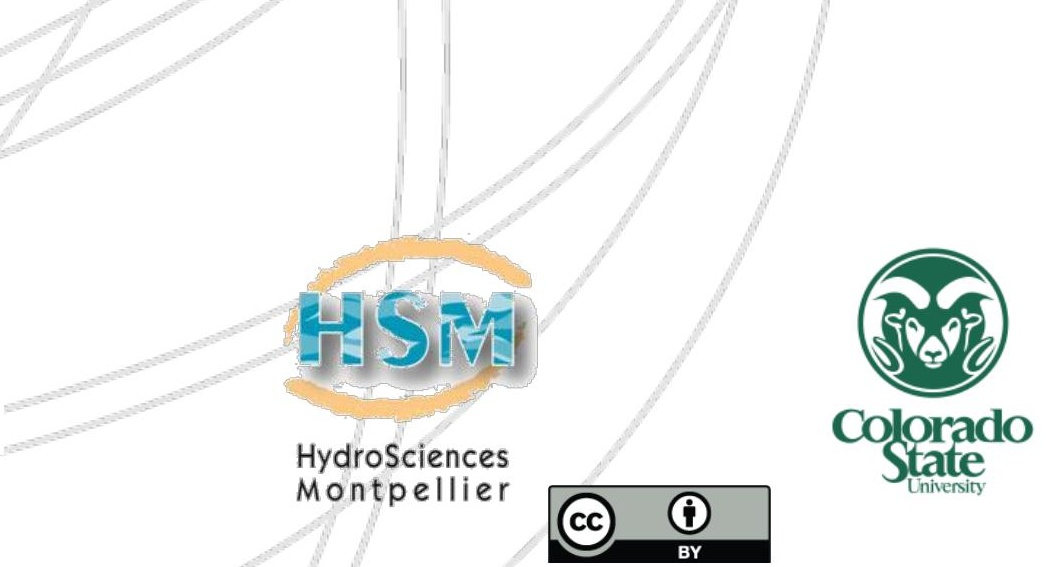


\section{CONTEXT}
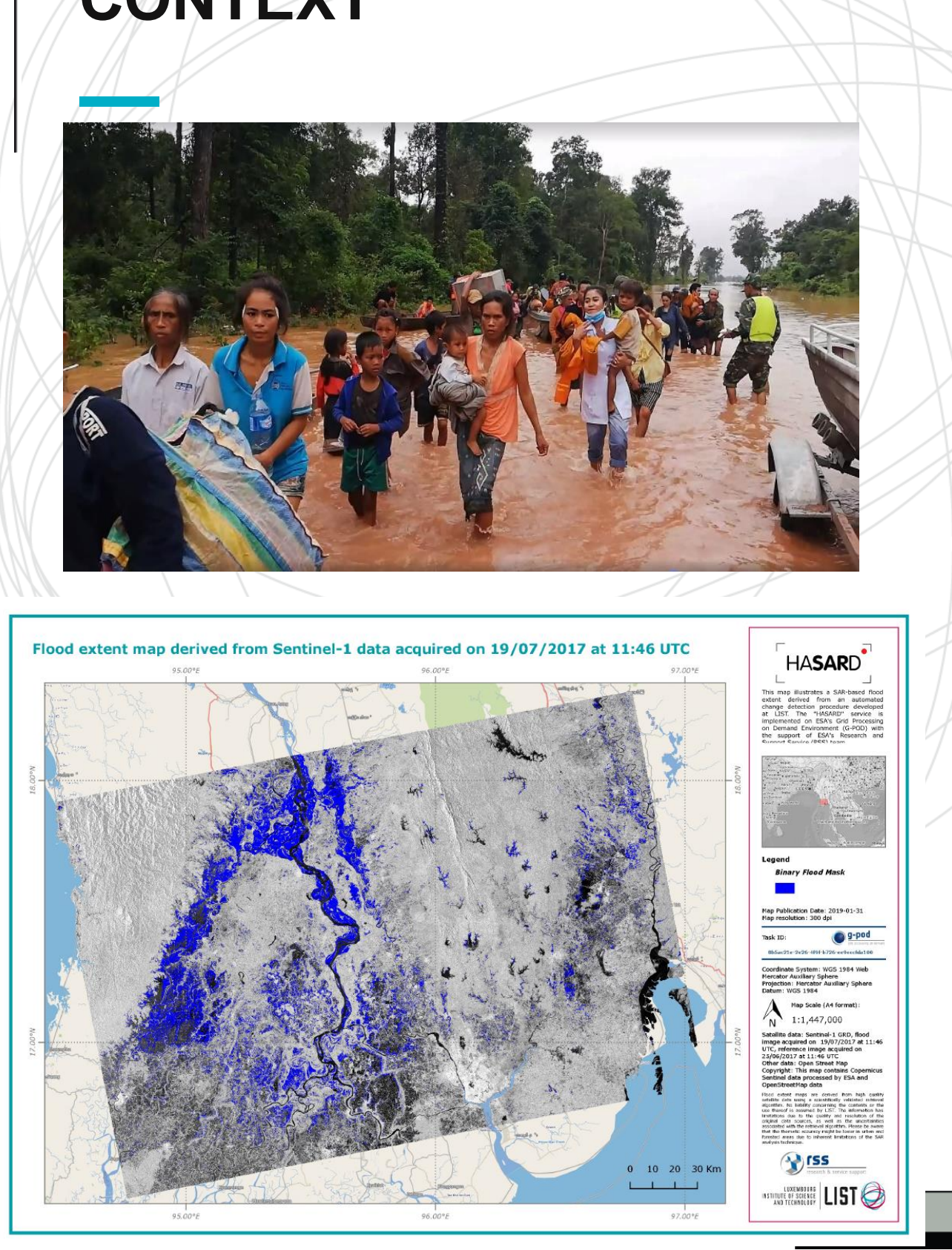

A powerful tool for flood management and prediction: hydrological modelling

Need for observations to set up, calibrate and evaluate these models. Issues:

$>$ Traditional observations are punctual ( $\mathrm{pb}$ of representativeness).

$>$ Observations are scarcely distributed and observation networks tend to be further reduced (e.g. stream gauges)

$>$ Ground observations not always reliable during flood events.

=> Need for new observation techniques: good candidates: satellite SAR flood images, and satellite derived soil moisture products 


\section{MORE AND MORE READILY AVAILABLE (RADAR) OBSERVATIONS}

\section{CeSa grid processing on demand}

Day-Night-All weather acquisitions

Synoptic view of large areas

Index differences between cdfs: 6.3211

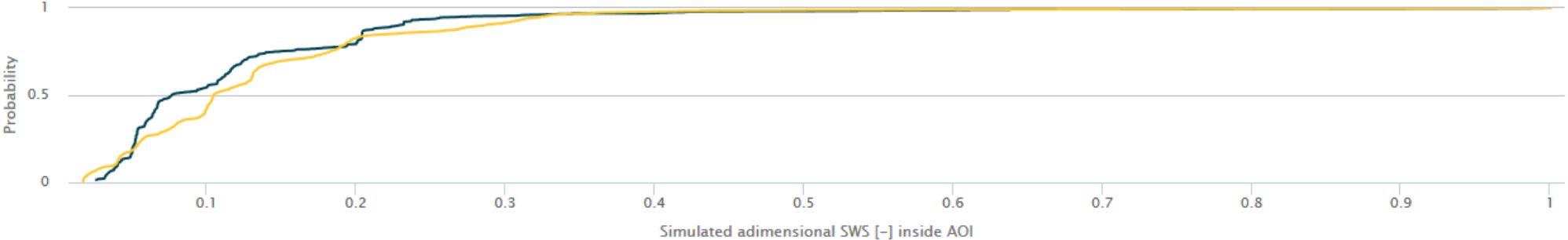

$\bullet$ Simulated SWS continuous time series $\rightarrow$ Simulated SWS at satellite acquisitions

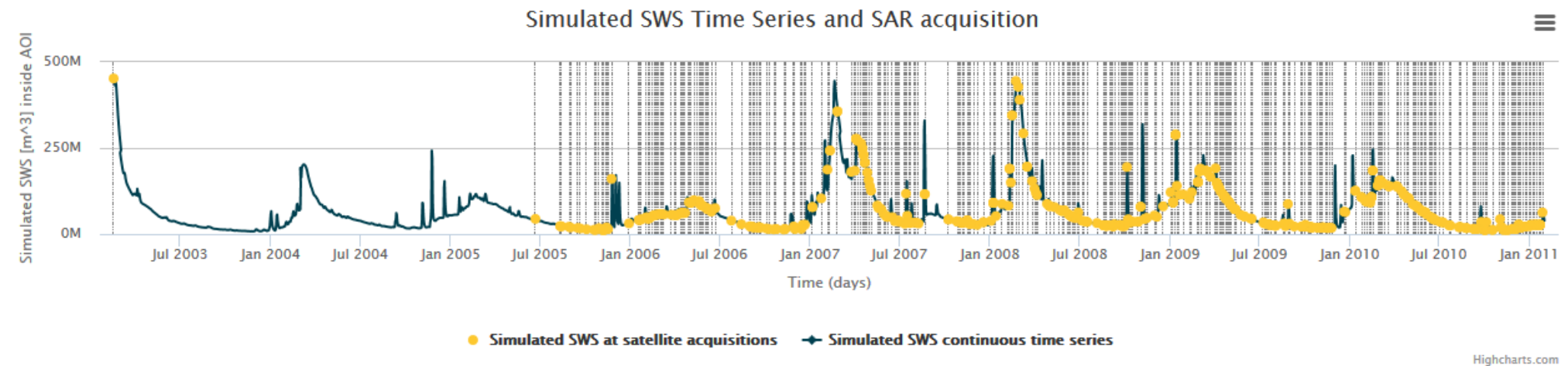




\section{MORE AND MORE READILY AVAILABLE (RADAR) OBSERVATIONS}

\section{CeSa grid processing on demand}

Day-Night-All weather acquisitions

Synoptic view of large areas

Index differences between cdfs: 6.3211

Flood extent Maps

$\rightarrow$ e.g. Sentinel- 1 (every 2-3 days over Europe, 6-12 days globally)
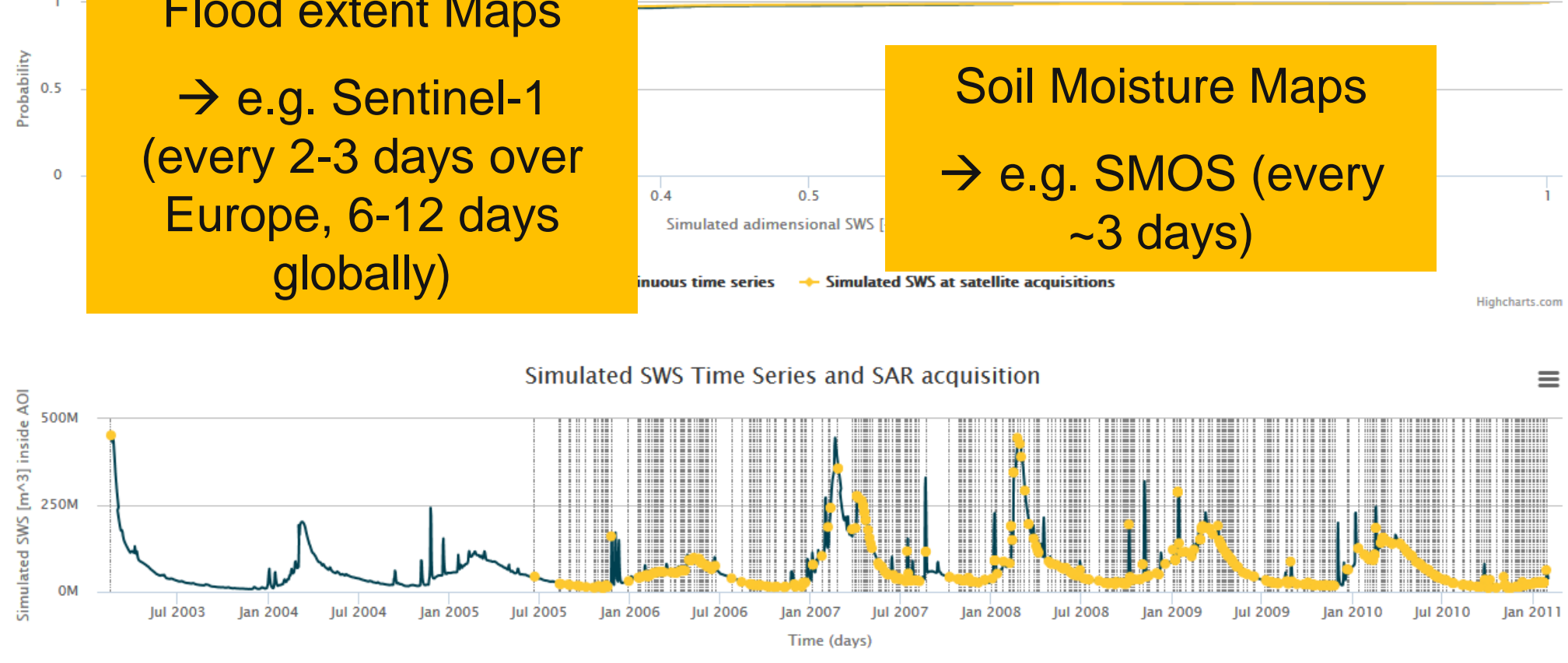

- Simulated SWS at satellite acquisitions $\rightarrow$ Simulated SWS continuous time series 


\section{THE CONCEPTUAL HYDROLOGICAL MODEL}




\section{THE MODEL STRUCTURE}

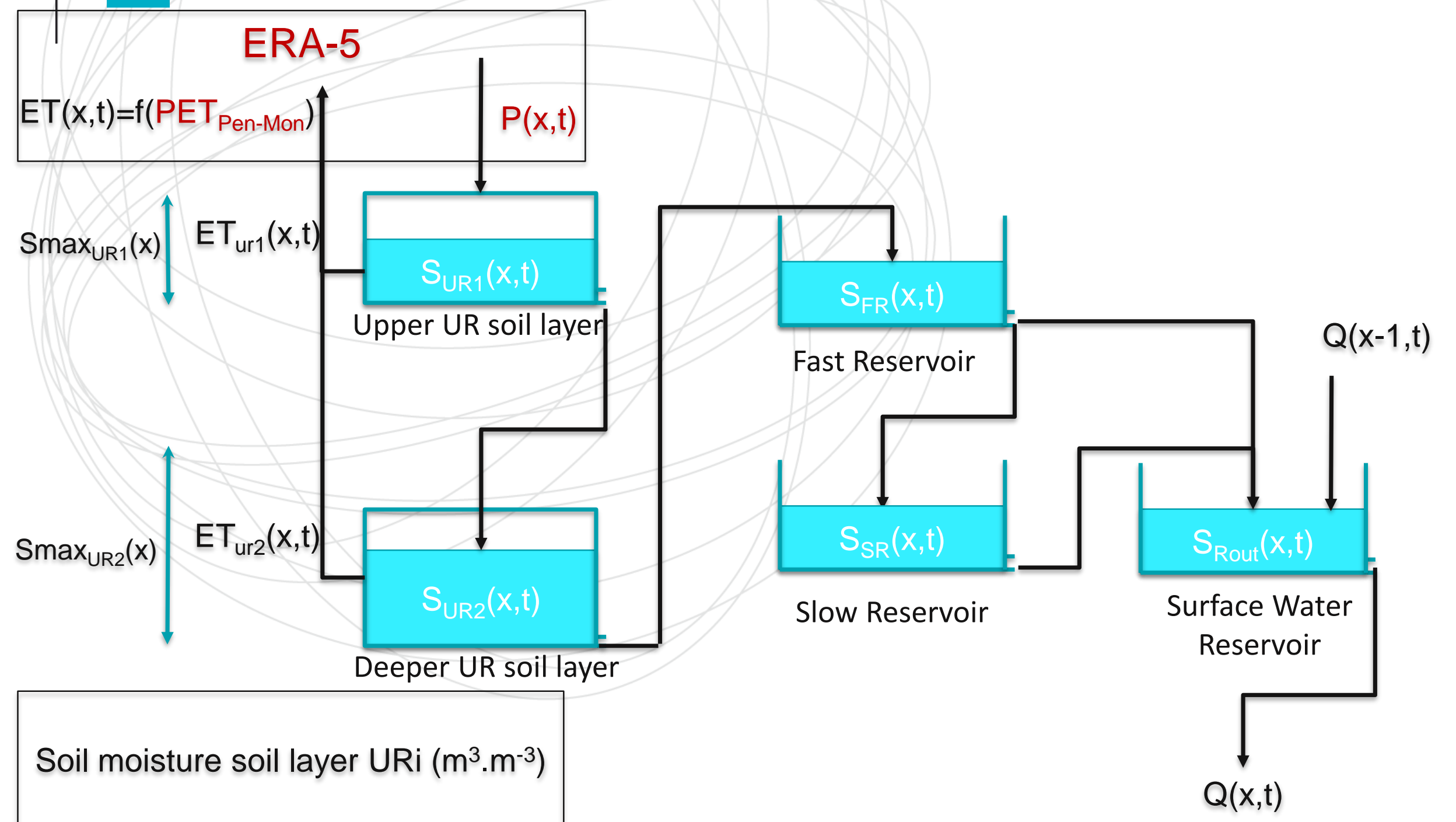

$$
S M_{u r i}=E F C_{u r i} * S_{u r i} / S m a x_{u r i}
$$




\section{THE MODEL STRUCTURE}

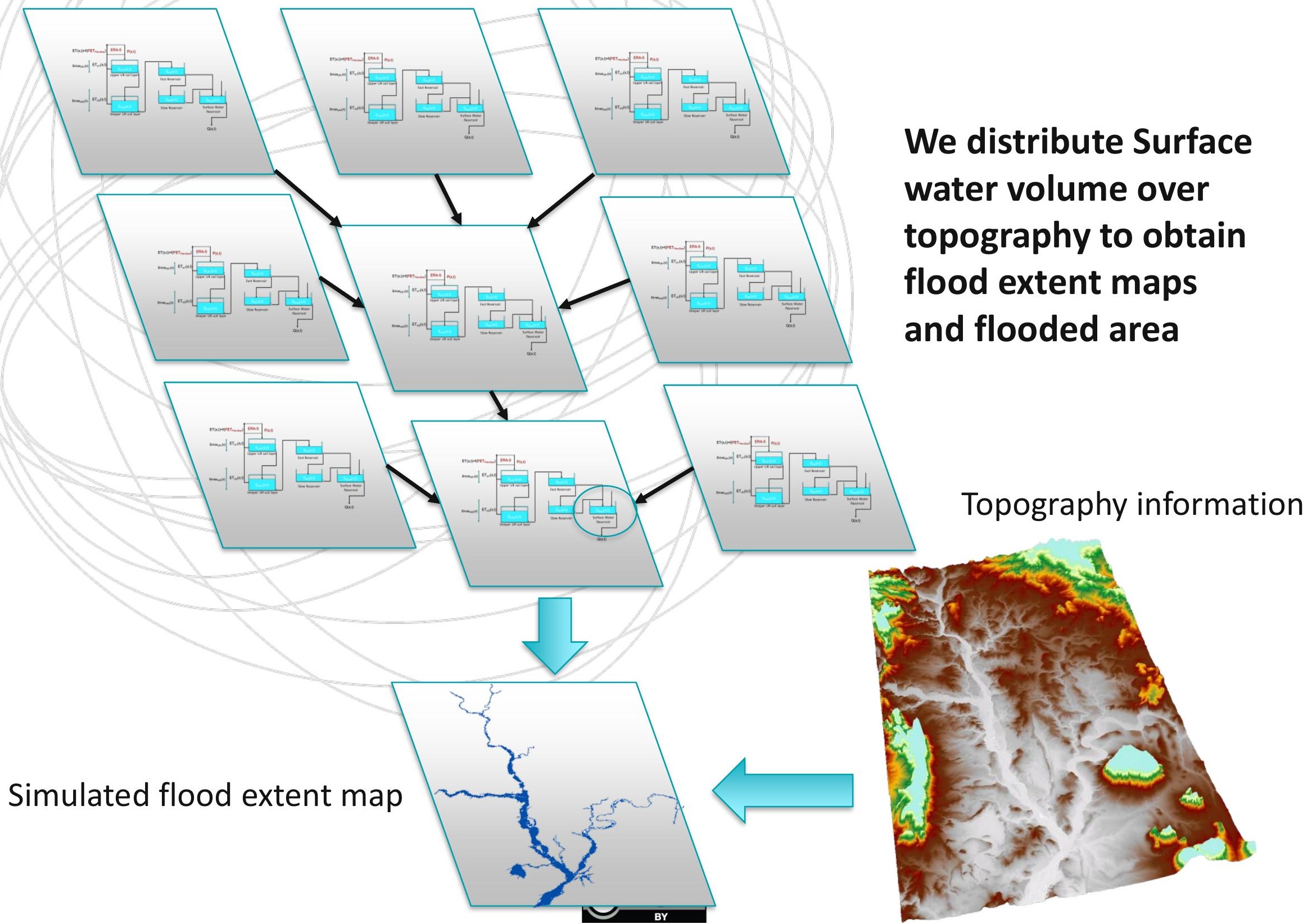




\section{ASSIMILATION DESIGN}




\section{ASSIMILATION DESIGN: THE OBSERVATION}

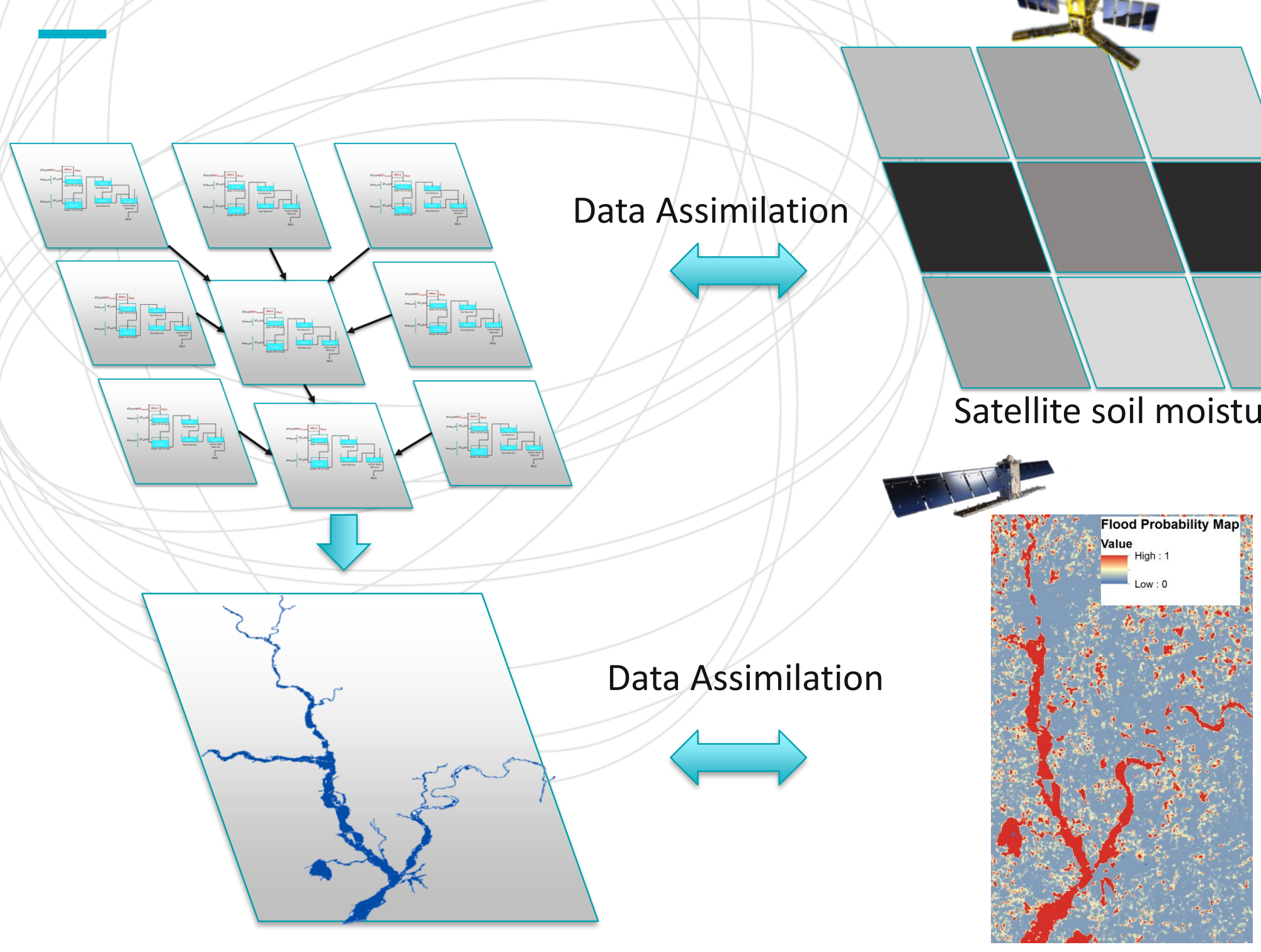




\section{THE ASSIMILATION DESIGN}

PARAMETER UPDATING STRATEGY

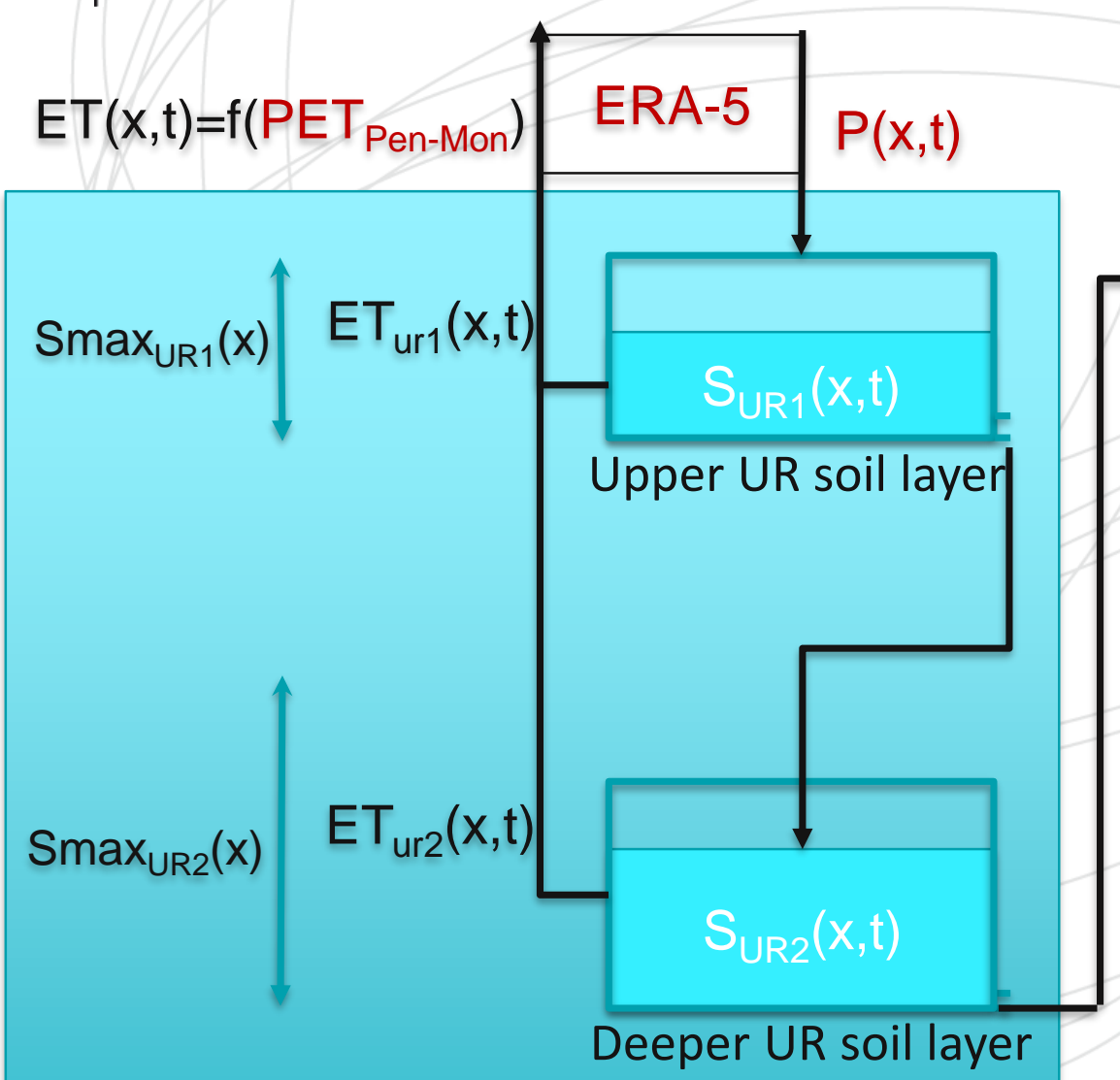

Soil Moisture Observation

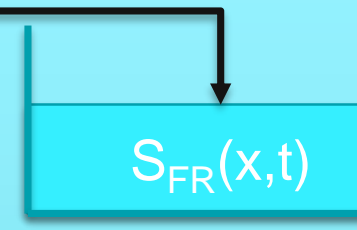

Fast Reservoir

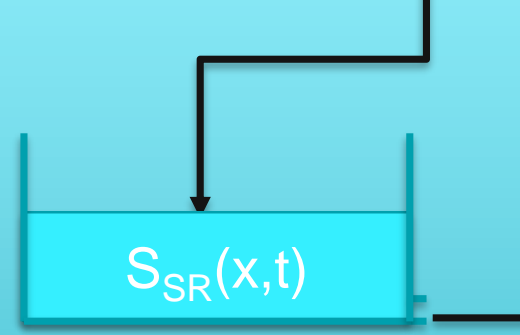

Slow Reservoir

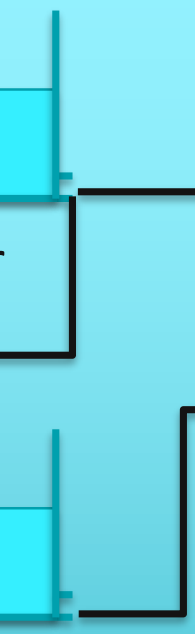

$\mathrm{Q}(\mathrm{x}-1, \mathrm{t})$

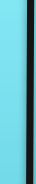

Surface Water Reservoir

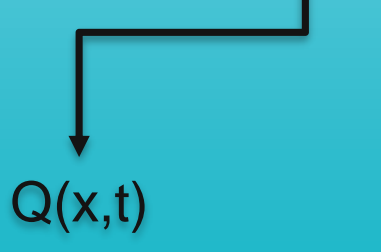

Flood Extent Observation 


\section{THE ASSIMILATION DESIGN:}

\section{A TEMPERED PARTICLE FILTER}

Bayes Theorem: $\quad \boldsymbol{p}(\theta \mid \boldsymbol{\theta})=\frac{\boldsymbol{p}(\boldsymbol{o} \mid \boldsymbol{\theta})}{\boldsymbol{p}(\boldsymbol{o})} \boldsymbol{p}(\boldsymbol{\theta})=\prod_{n=1}^{K} \frac{\boldsymbol{p}(\boldsymbol{o} \mid \boldsymbol{\theta})^{\varphi_{n}-\varphi_{n-1}}}{\boldsymbol{p}(\boldsymbol{o})} \boldsymbol{p}(\theta)$

$$
0=\varphi_{0}<\varphi_{1}<\varphi_{2}<\cdots<\varphi_{K}=1
$$
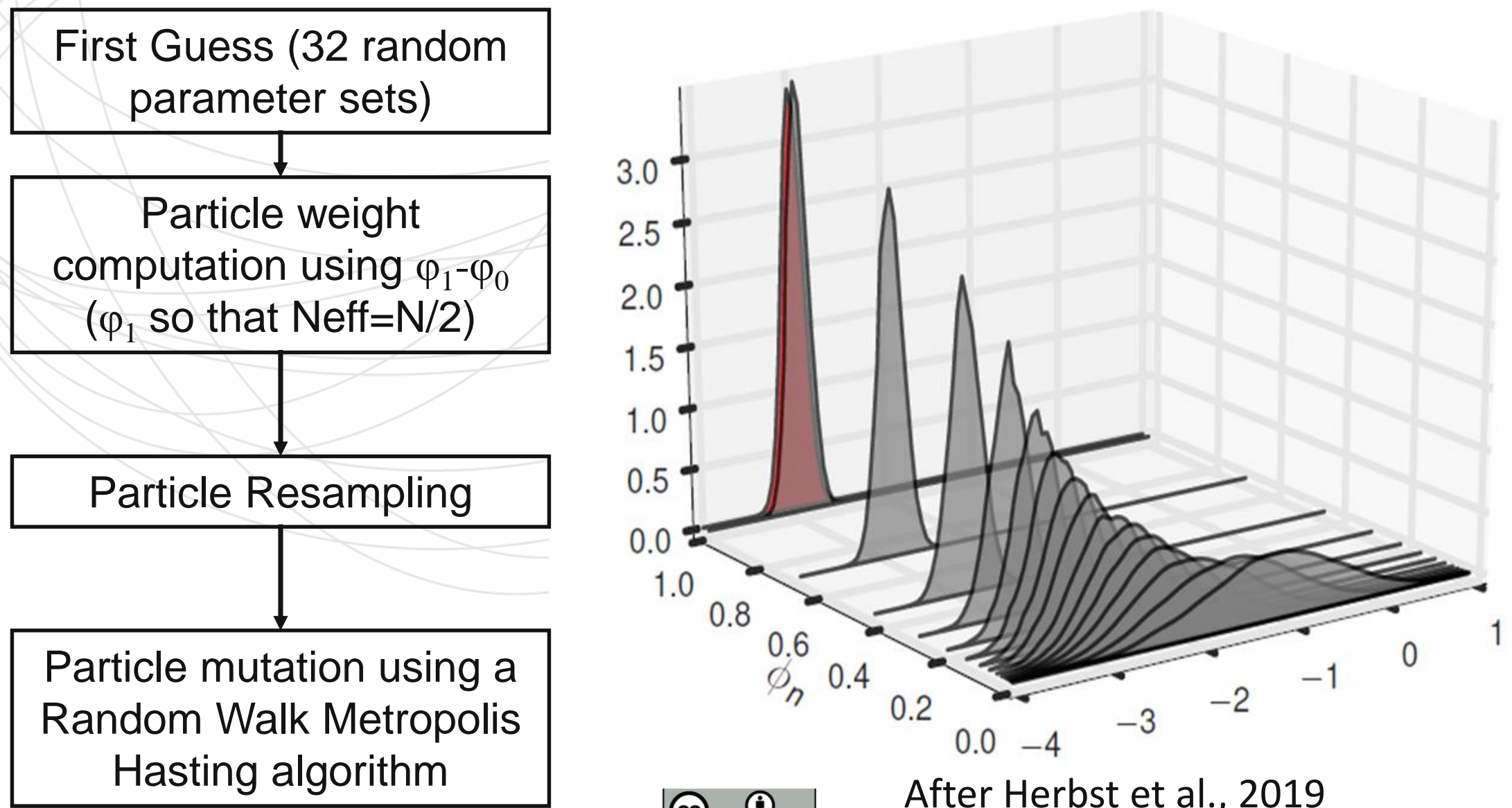

(c) (1) After Herbst et al., 2019 


\section{THE ASSIMILATION DESIGN:}

\section{A TEMPERED PARTICLE FILTER}

Bayes Theorem: $\quad \boldsymbol{p}(\theta \mid \boldsymbol{\theta})=\frac{\boldsymbol{p}(\boldsymbol{o} \mid \boldsymbol{\theta})}{\boldsymbol{p}(\boldsymbol{o})} \boldsymbol{p}(\boldsymbol{\theta})=\prod_{n=1}^{K} \frac{\boldsymbol{p}(\boldsymbol{o} \mid \boldsymbol{\theta})^{\varphi_{n}-\varphi_{n-1}}}{\boldsymbol{p}(\boldsymbol{o})} \boldsymbol{p}(\theta)$

$$
0=\varphi_{0}<\varphi_{1}<\varphi_{2}<\cdots<\varphi_{K}=1
$$

First Guess (32 random parameter sets)

Particle weight

$\longrightarrow$ computation using $\varphi_{n}-\varphi_{n-1}$ $\left(\varphi_{\mathrm{n}}\right.$ so that $\left.\mathrm{Neff}=\mathrm{N} / 2\right)$

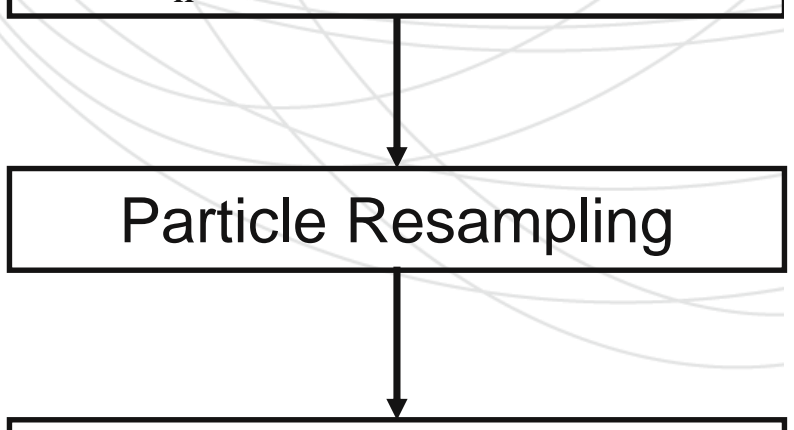

Particle mutation using a Random Walk Metropolis Hasting algorithm

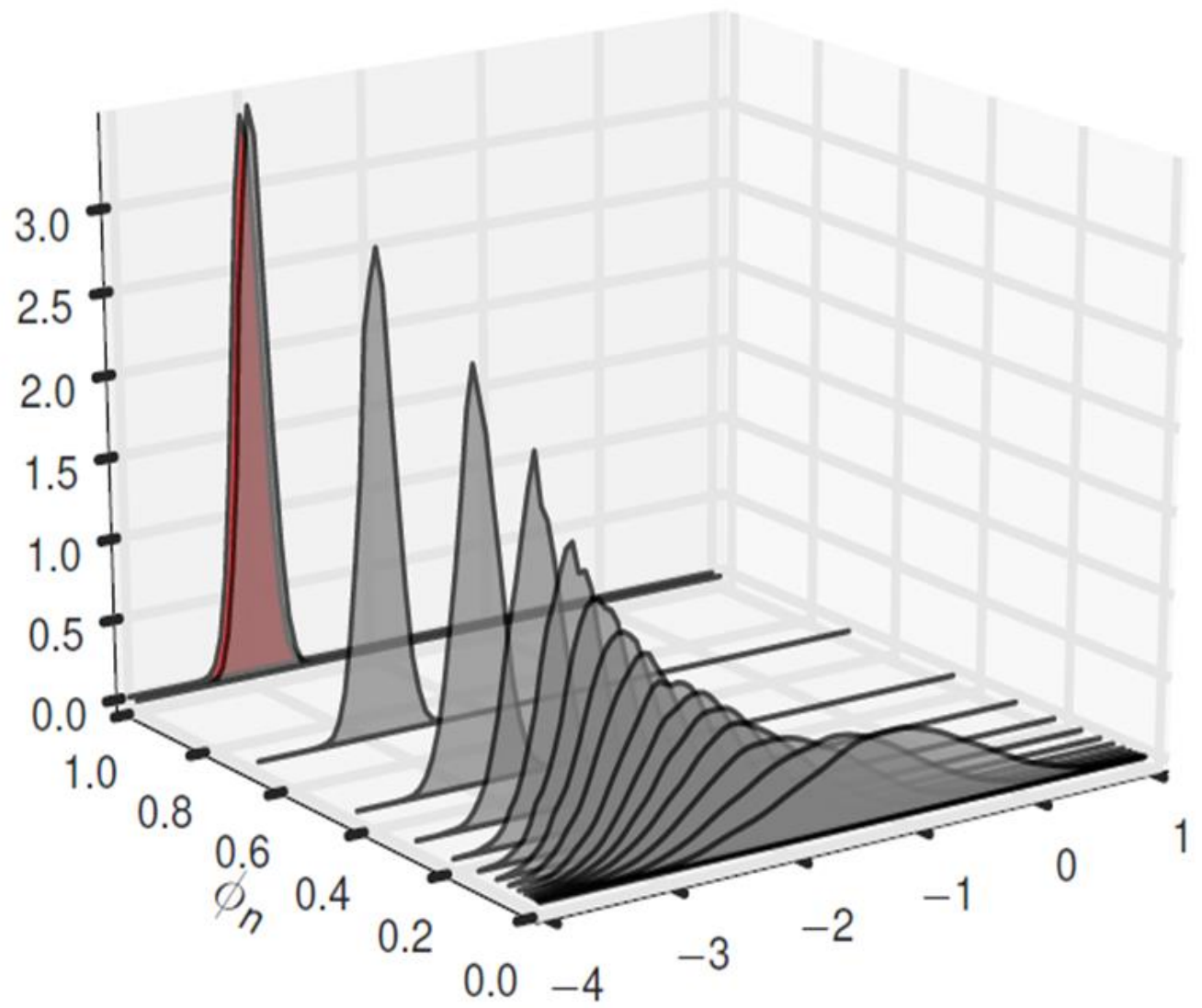

(c) (1) After Herbst et al., 2019 


\section{SYNTHETIC TWIN EXPERIMENTS}




\section{SYNTHETIC TWIN EXPERIMENTS: SYNTHETIC TRUTH AND OBSERVATION}

\section{Model forward run (9 years, hourly)}

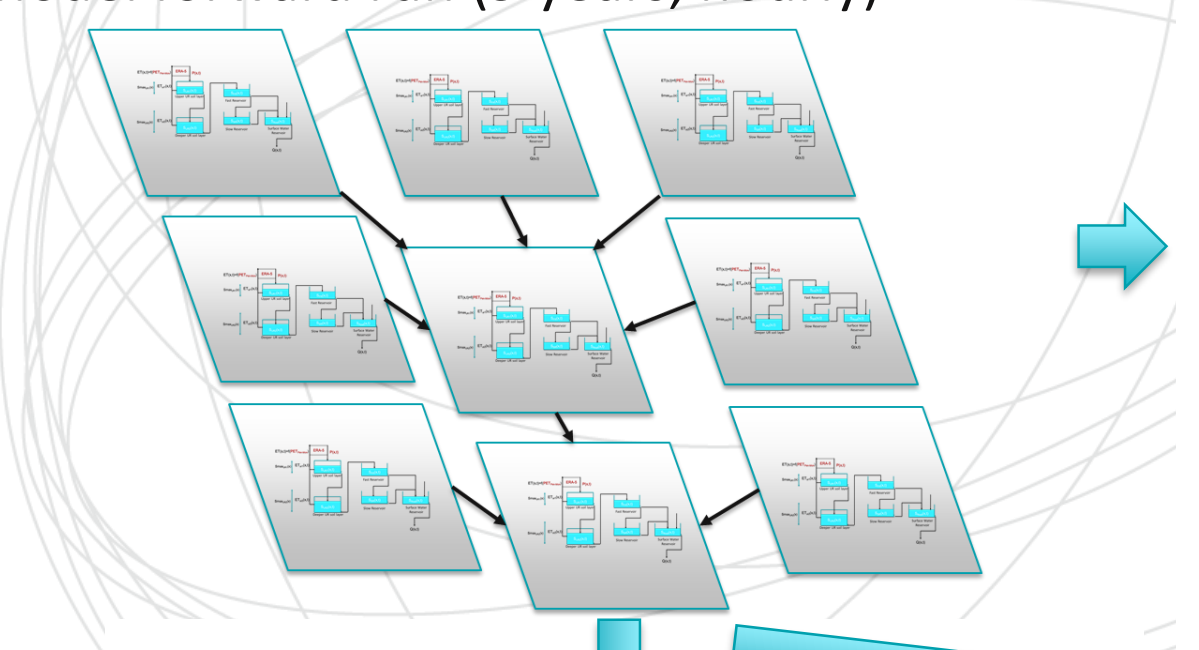

Soil moisture (SM): One Obs. every 3 days

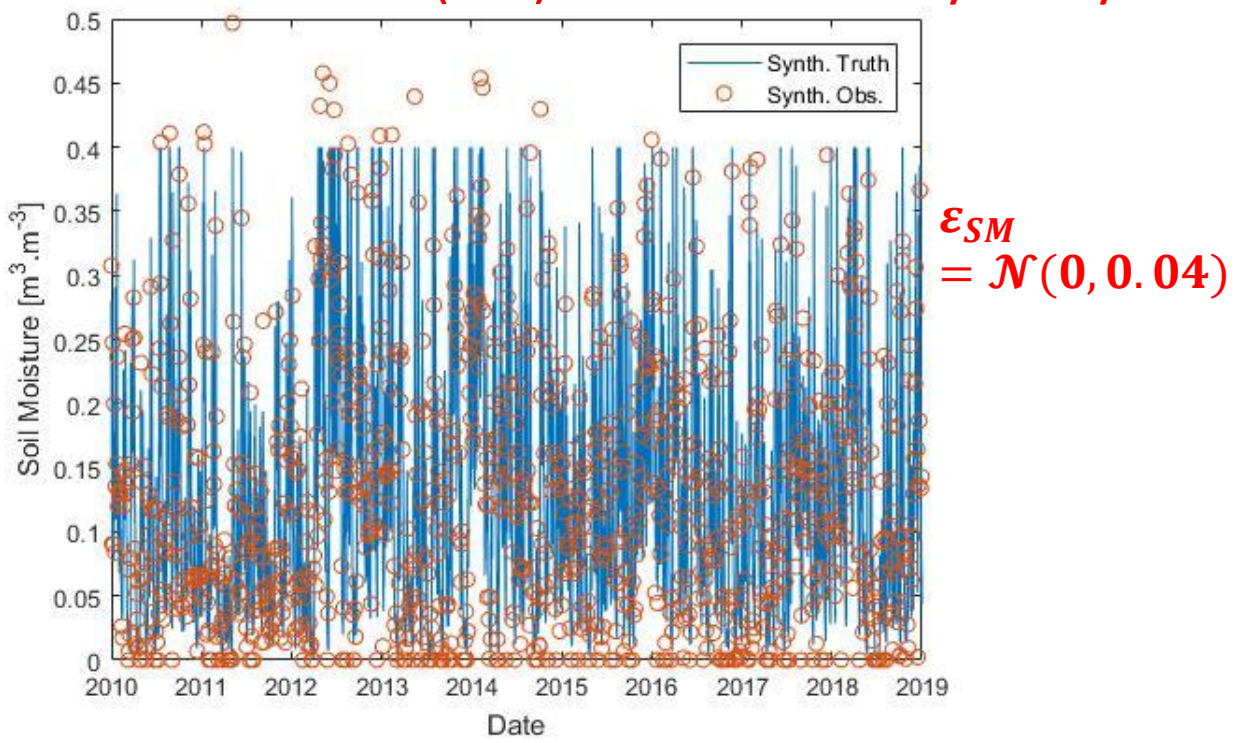

Flooded Area (FA) One $\mathrm{Ob}^{50}$. every 3 days

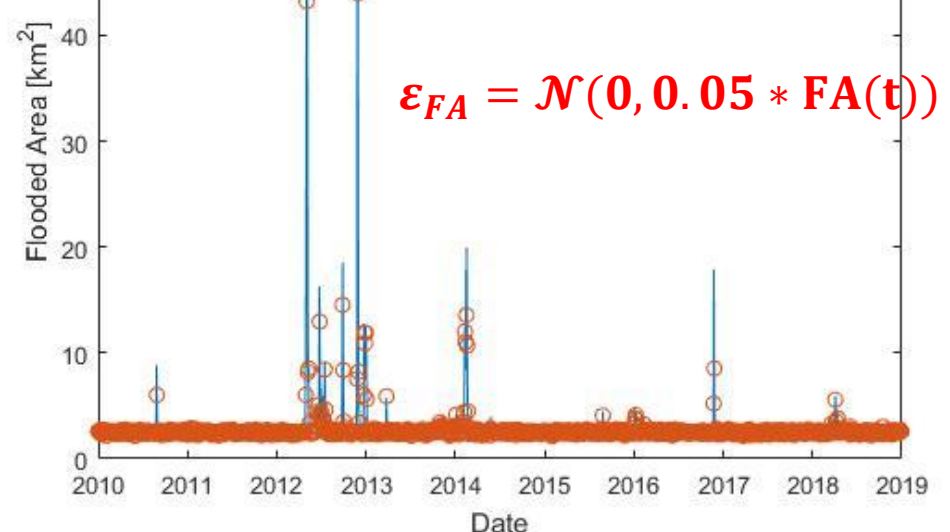

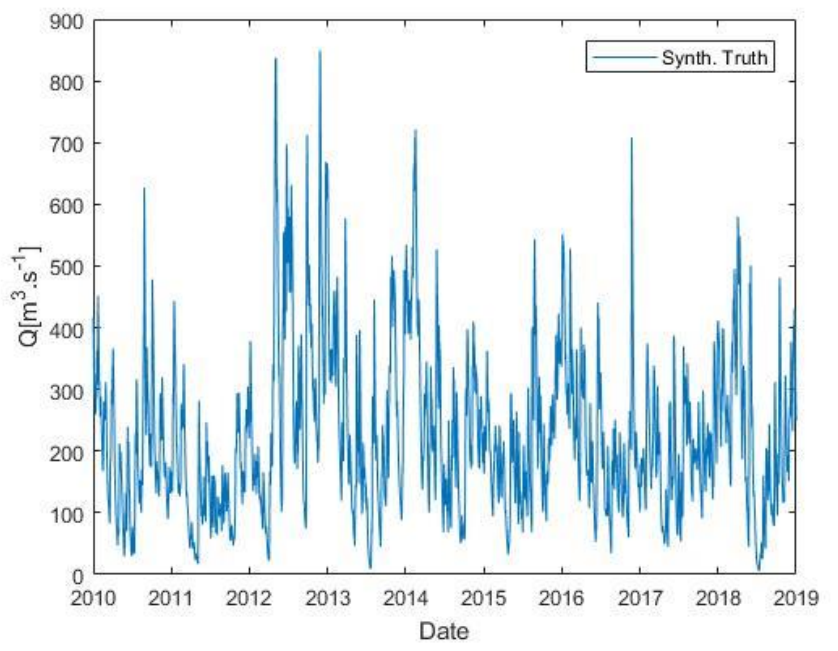




\section{SYNTHETIC TWIN EXPERIMENTS: MODEL CALIBRATION USING SM+FA}

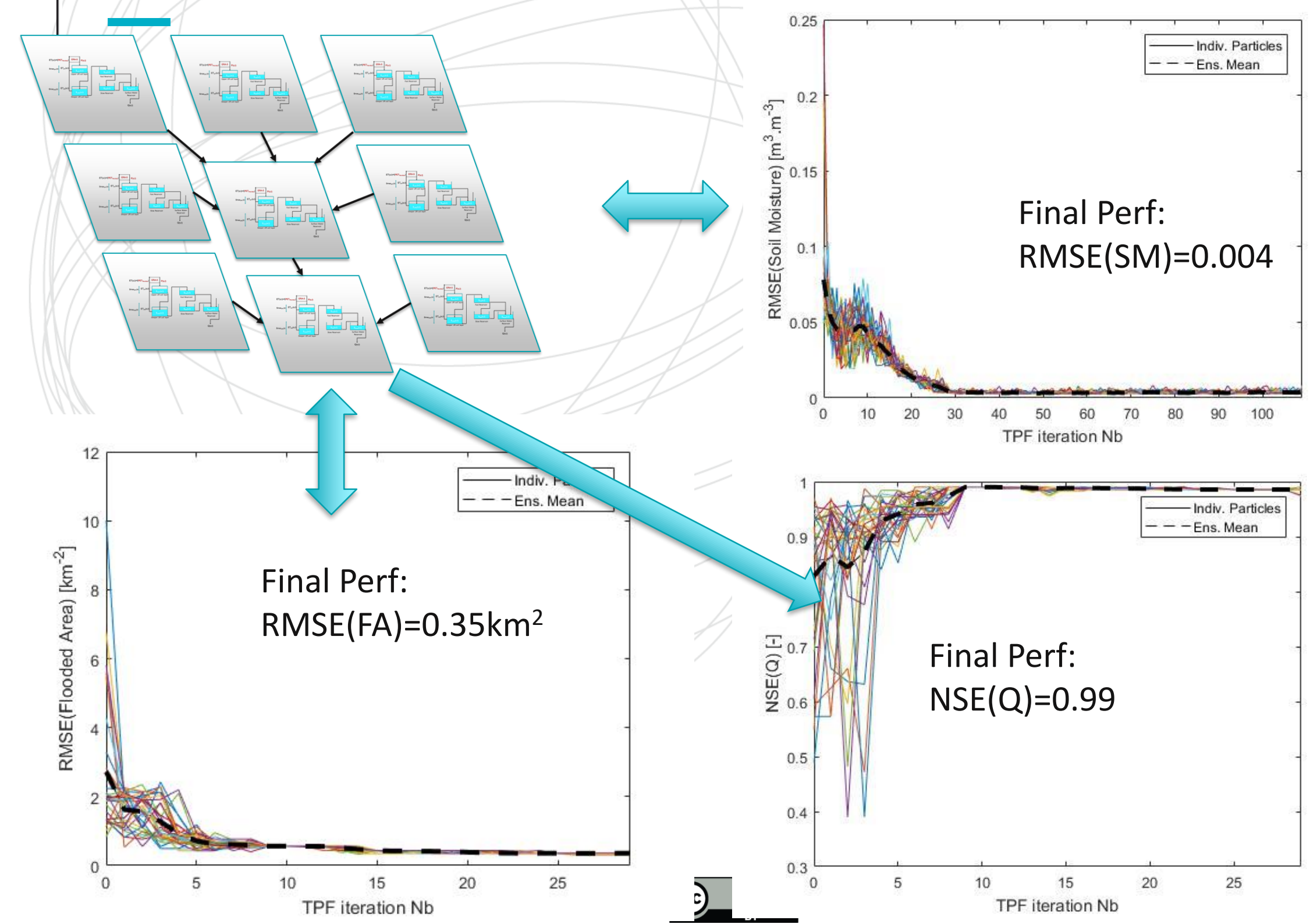




\section{SYNTHETIC TWIN EXPERIMENTS: CALIBRATED MODEL EVALUATION}

\section{$\mathrm{SM}+\mathrm{FA}$}
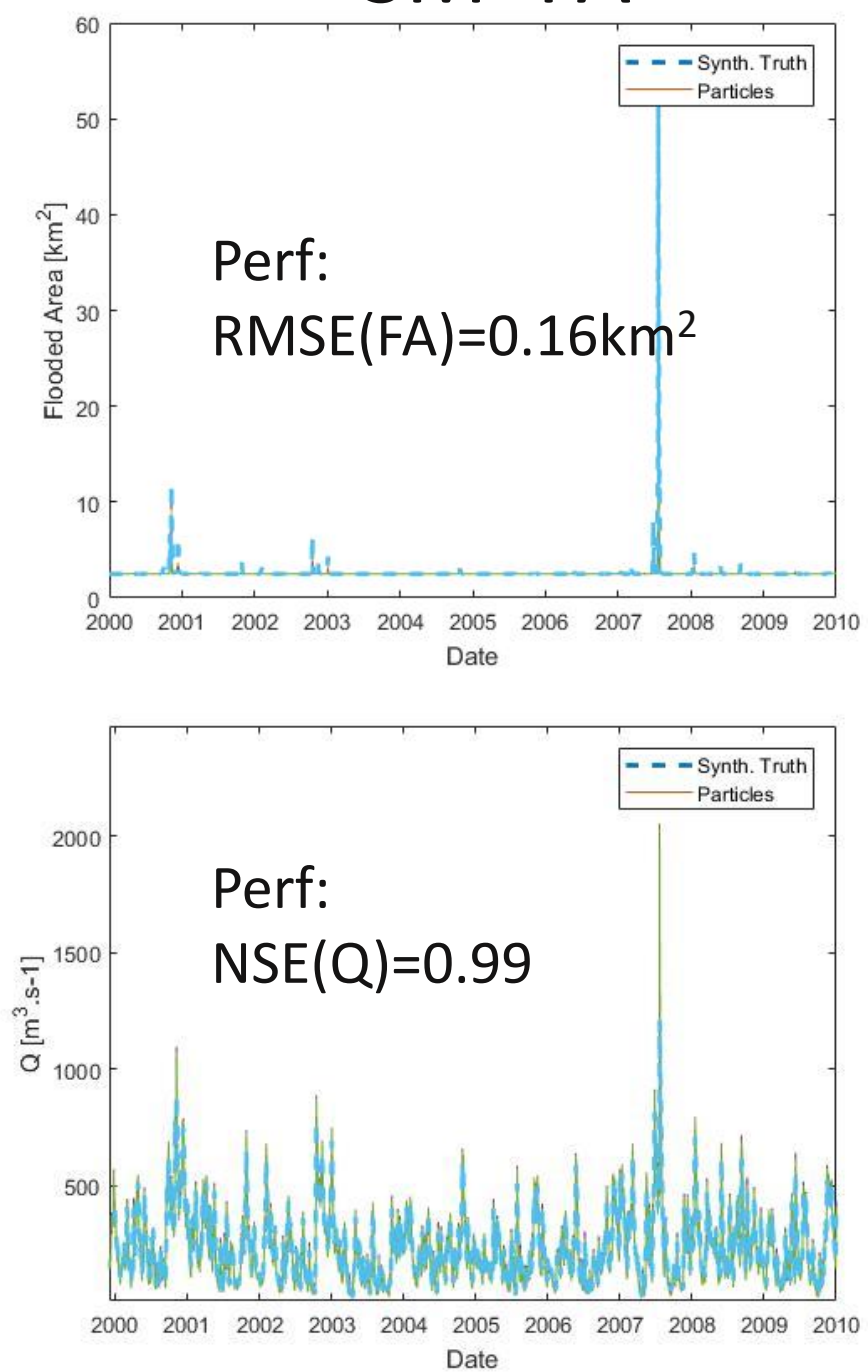
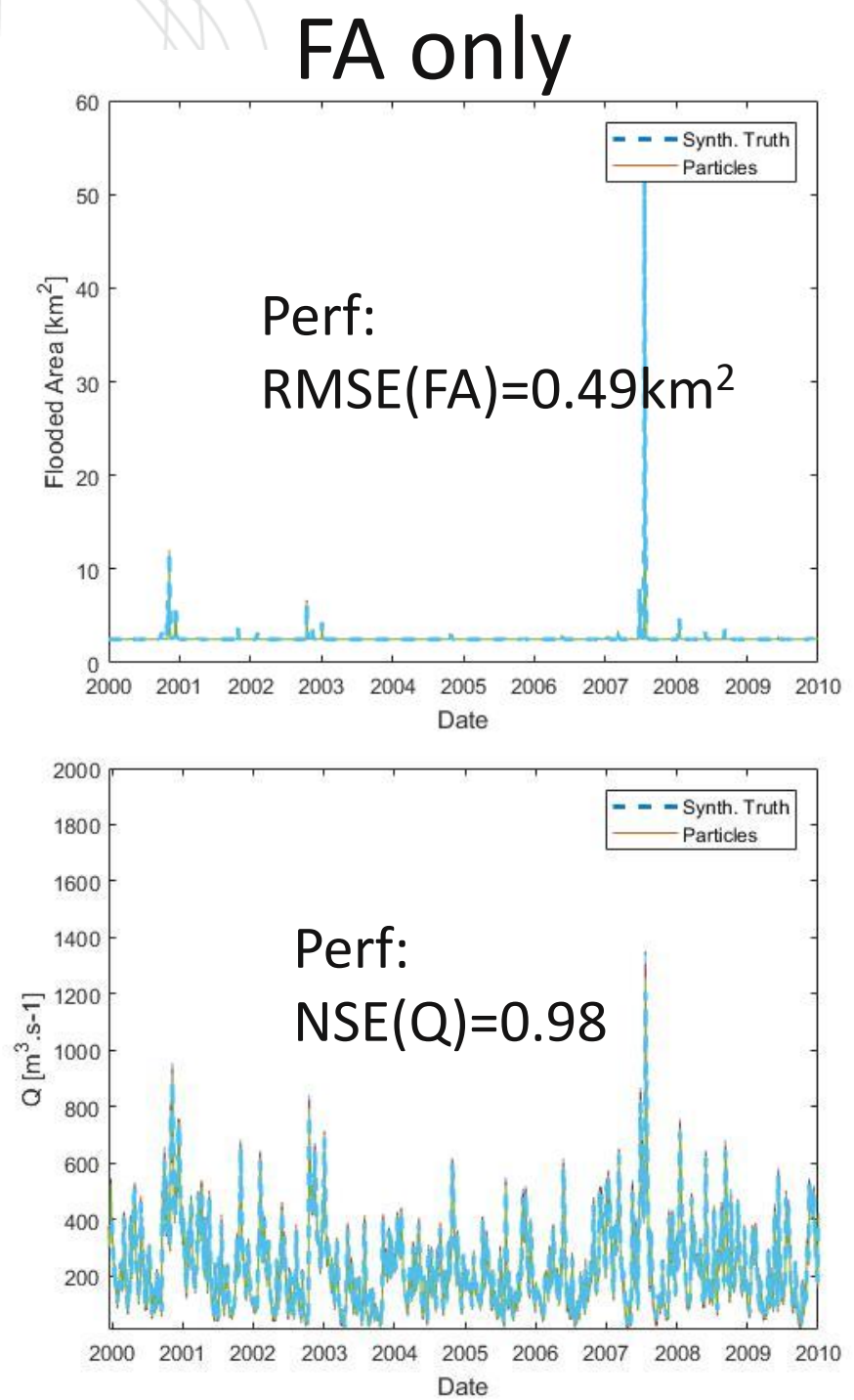


\section{CONCLUSION \& NEXT STEPS}

- We carried out a synthetic experiment using a TPF of the joint assimilation of satellite flooded area and soil moisture observation

- The results are really promising as the calibrated model is predicting surface runoff accurately both during the calibration and the validation periods

- This opens the floor for applications at large scale over poorly gauged areas

Next steps:

- To further investigate the added value of soil moisture data

- To carry out real test case experiments 


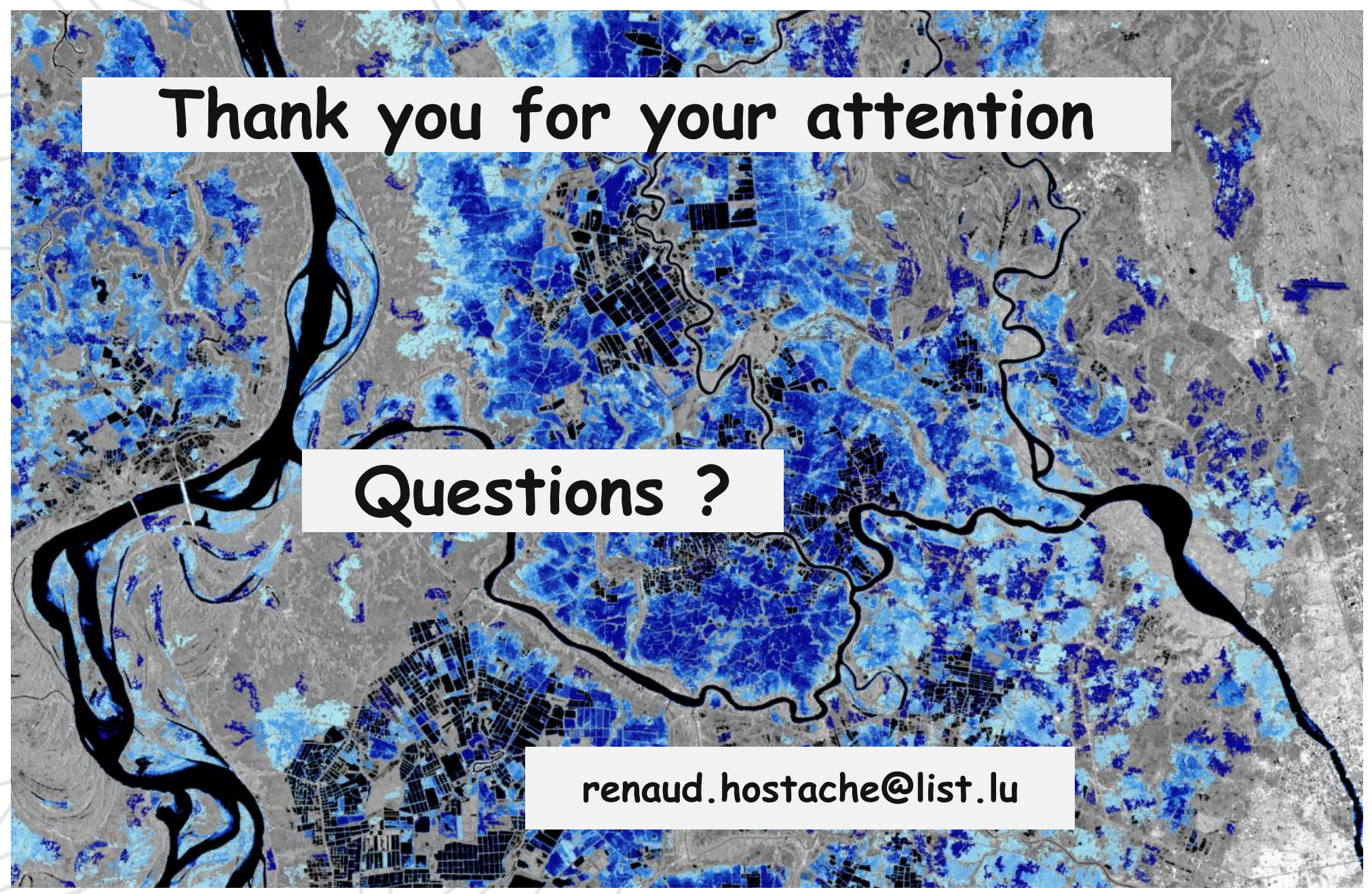

Acknowledgments: Research funded by the National Research Fund (FNR) of Luxembourg through the CASCADE project (grant C17/SR/11682050), and the NERC through the DARE project (EP/P002331/1). 